\title{
A TALE OF TWO ARC LENGTHS: METRIC NOTIONS FOR CURVES IN SURFACES IN EQUIAFFINE SPACE
}

\author{
JEANNE N. CLELLAND, EDWARD ESTRADA, MOLLY MAY, JONAH MILLER, \\ SEAN PENEYRA, AND MICHAEL SCHMIDT
}

(Communicated by Lei $\mathrm{Ni}$ )

\begin{abstract}
In Euclidean geometry, all metric notions (arc length for curves, the first fundamental form for surfaces, etc.) are derived from the Euclidean inner product on tangent vectors, and this inner product is preserved by the full symmetry group of Euclidean space (translations, rotations, and reflections). In equiaffine geometry, there is no invariant notion of inner product on tangent vectors that is preserved by the full equiaffine symmetry group. Nevertheless, it is possible to define an invariant notion of arc length for nondegenerate curves and an invariant first fundamental form for nondegenerate surfaces in equiaffine space. This leads to two possible notions of arc length for a curve contained in a surface, and these two arc length functions do not necessarily agree. In this paper we will derive necessary and sufficient conditions under which the two arc length functions do agree, and illustrate with examples.
\end{abstract}

\section{INTRODUCTION}

The primary defining characteristic of Euclidian geometry in $\mathbb{R}^{3}$ is the presence of a flat metric $\langle$,$\rangle which is defined on all tangent vectors to all points of \mathbb{R}^{3}$ and invariant under the action of the Euclidean group. When studying submanifolds of the Euclidean space $\mathbb{E}^{3}$ (i.e., $\mathbb{R}^{3}$ together with a Euclidean metric), all metric properties (e.g., arc lengths, surface areas) are derived from this underlying metric. By contrast, in equiaffine geometry (which, for convenience, we will refer to simply as "affine geometry"), it is not possible to define a metric on tangent vectors which is preserved by the action of the equiaffine group. There is an invariant volume form, but no invariant notion of distance which can be restricted to submanifolds of $\mathbb{A}^{3}$ (i.e., $\mathbb{R}^{3}$ together with an equiaffine structure) in any obvious way.

Nevertheless, it is possible to define a notion of affine arc length for generic curves in affine space, as well as a notion of an affine metric for generic surfaces, in such a way that these notions are preserved by the action of the equiaffine group. Because there is no inner product on tangent vectors, these affine notions of metrics on submanifolds depend on higher-order derivatives, as opposed to the analogous Euclidean notions, which depend only on first derivatives of the submanifolds in question.

Now, suppose that we have a curve $\alpha$ contained in a surface $\Sigma \subset \mathbb{A}^{3}$. The affine metric on $\Sigma$ can be restricted to $\alpha$ in order to define an arc length function on $\alpha$.

Received by the editors May 1, 2012 and, in revised form, August 8, 2012.

2010 Mathematics Subject Classification. Primary 53A15, 53A55; Secondary 53A04, 53A05.

This research was supported in part by NSF grant DMS-0908456. 
Unlike in Euclidean geometry, it is possible for this arc length function to differ from the affine arc length function on $\alpha$ considered as a curve in $\mathbb{A}^{3}$. The goal of this paper is to explore these two different notions of affine arc length for $\alpha$ and to consider conditions under which they may agree.

The remainder of the paper is structured as follows. In $\$ 2$ we will recall the definitions of affine arc length for nondegenerate curves in $\mathbb{A}^{3}$ and the affine first fundamental form for nondegenerate surfaces in $\mathbb{A}^{3}$. In $₫ 3$ we will explore how these two notions give rise to two different notions of arc length for a curve $\alpha \subset \Sigma \subset \mathbb{A}^{3}$, and we will construct several examples where these notions do not agree. In 4 , we will determine conditions under which these two notions do agree, and in 95 , we will construct examples of curves on surfaces for which the two notions of arc length are the same.

\section{Notions FROM AFFine GEOMETRY}

2.1. The Euclidean group and the equiaffine group. Three-dimensional Euclidean space $\mathbb{E}^{3}$ consists of the vector space $\mathbb{R}^{3}$, together with a positive definite inner product

$$
\langle\cdot, \cdot\rangle: \mathbb{R}^{3} \times \mathbb{R}^{3} \rightarrow \mathbb{R}
$$

The Euclidean group $E(3)$ is the group of all transformations $\phi: \mathbb{E}^{3} \rightarrow \mathbb{E}^{3}$ which preserve the inner product structure; it consists of all transformations of the form

$$
\phi(\mathbf{x})=A \mathbf{x}+\mathbf{b},
$$

where $A \in O(3)$ and $\mathbf{b} \in \mathbb{E}^{3}$.

Three-dimensional equiaffine space $\mathbb{A}^{3}$ (which for convenience we will refer to simply as "affine space") consists of the vector space $\mathbb{R}^{3}$, together with a nondegenerate volume form

$$
d V: \Lambda^{3} \mathbb{R}^{3} \rightarrow \mathbb{R}
$$

The equiaffine group $A(3)$ is the group of all transformations $\phi: \mathbb{E}^{3} \rightarrow \mathbb{E}^{3}$ which preserve the volume form; it consists of all transformations of the form

$$
\phi(\mathbf{x})=A \mathbf{x}+\mathbf{b},
$$

where $A \in S L(3)$ and $\mathbf{b} \in \mathbb{A}^{3}$.

Note that, while the inner product structure on $\mathbb{E}^{3}$ induces a volume form on $\mathbb{E}^{3}$, the converse is false: there is no inner product on $\mathbb{A}^{3}$ which is preserved by the action of the equiaffine group $A(3)$. Thus in equiaffine geometry there are no obvious analogs of metric notions such as length or angles defined on tangent vectors.

2.2. Curves and affine arc length. Let $I \subset \mathbb{R}$, and let $\alpha: I \rightarrow \mathbb{R}^{3}$ be a regular curve. ( $\alpha$ may be considered as a curve in either $\mathbb{E}^{3}$ or $\mathbb{A}^{3}$.) In Euclidean geometry, one generally associates to $\alpha$ the Frenet frame $\left(\mathbf{e}_{1}, \mathbf{e}_{2}, \mathbf{e}_{3}\right)$ defined by

$$
\mathbf{e}_{1}(t)=\frac{\alpha^{\prime}(t)}{\left\|\alpha^{\prime}(t)\right\|}, \quad \mathbf{e}_{2}(t)=\frac{\mathbf{e}_{1}^{\prime}(t)}{\left\|\mathbf{e}_{1}^{\prime}(t)\right\|}, \quad \mathbf{e}_{3}(t)=\mathbf{e}_{1}(t) \times \mathbf{e}_{2}(t),
$$

where for any vector $\mathbf{v}$, we define $\|\mathbf{v}\|=\sqrt{\langle\mathbf{v}, \mathbf{v}\rangle}$. (See, e.g., 3.) This frame is well-defined provided that the vectors $\alpha^{\prime}(t), \alpha^{\prime \prime}(t)$ are linearly independent for each 
$t \in I$; such a curve is called a nondegenerate curve in $\mathbb{E}^{3}$. The Euclidean arc length function $\bar{s}(t)$ of $\alpha$ is defined by

$$
\bar{s}(t)=\int_{0}^{t} \sqrt{\left\langle\alpha^{\prime}(\sigma), \alpha^{\prime}(\sigma)\right\rangle} d \sigma .
$$

It has the property that if $\alpha$ is reparametrized via the inverse function $t(\bar{s})$ as

$$
\alpha(\bar{s})=\alpha(t(\bar{s})),
$$

then $\left\|\alpha^{\prime}(\bar{s})\right\| \equiv 1$, and so the Frenet frame satisfies $\mathbf{e}_{1}(\bar{s})=\alpha^{\prime}(\bar{s})$. Moreover, the Frenet frame satisfies the Frenet equations

$$
\left[\begin{array}{lll}
\mathbf{e}_{1}^{\prime}(\bar{s}) & \mathbf{e}_{2}^{\prime}(\bar{s}) & \mathbf{e}_{3}^{\prime}(\bar{s})
\end{array}\right]=\left[\begin{array}{lll}
\mathbf{e}_{1}(\bar{s}) & \mathbf{e}_{2}(\bar{s}) & \mathbf{e}_{3}(\bar{s})
\end{array}\right]\left[\begin{array}{ccc}
0 & -\kappa(\bar{s}) & 0 \\
\kappa(\bar{s}) & 0 & \tau(\bar{s}) \\
0 & -\tau(\bar{s}) & 0
\end{array}\right],
$$

where $\kappa(\bar{s}), \tau(\bar{s})$ are the curvature and torsion functions, respectively, of $\alpha$. An

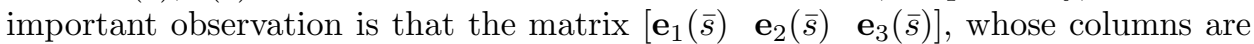
the Frenet frame vectors, is an element of the Lie group $S O(3)$, which is precisely the (oriented) symmetry group of the Euclidean structure on each tangent space.

Now suppose that we consider $\alpha: I \rightarrow \mathbb{R}^{3}$ as a curve in $\mathbb{A}^{3}$. We no longer have the notions of vector norm and cross product to make use of in order to define a frame along the curve. But if we define

$$
\mathbf{e}_{1}(t)=\alpha^{\prime}(t), \quad \mathbf{e}_{2}(t)=\alpha^{\prime \prime}(t), \quad \mathbf{e}_{3}(t)=\alpha^{\prime \prime \prime}(t),
$$

then the quantity

$$
\operatorname{det}\left[\begin{array}{lll}
\mathbf{e}_{1}(t) & \mathbf{e}_{2}(t) & \mathbf{e}_{3}(t)
\end{array}\right]
$$

is invariant under the action of the equiaffine group. By way of analogy with the Euclidean case, it would be nice to find a parametrization of the curve for which the matrix $\left[\begin{array}{lll}\mathbf{e}_{1}(t) & \mathbf{e}_{2}(t) & \mathbf{e}_{3}(t)\end{array}\right]$ is an element of the symmetry group $S L(3)$, i.e., for which

$$
\operatorname{det}\left[\begin{array}{lll}
\mathbf{e}_{1}(t) & \mathbf{e}_{2}(t) & \mathbf{e}_{3}(t)
\end{array}\right]=1 .
$$

This motivation leads to the following definitions (see, e.g., [7]):

- A curve $\alpha: I \rightarrow \mathbb{A}^{3}$ is called nondegenerate if the vectors $\alpha^{\prime}(t), \alpha^{\prime \prime}(t), \alpha^{\prime \prime \prime}(t)$ are linearly independent for each $t \in I$. (Note that this is different from the Euclidean definition.)

- The affine arc length function $s_{\alpha}(t)$ of a nondegenerate curve $\alpha$ is defined by

$$
s_{\alpha}(t)=\int_{0}^{t} \sqrt[6]{\operatorname{det}\left[\alpha^{\prime}(\sigma) \alpha^{\prime \prime}(\sigma) \alpha^{\prime \prime \prime}(\sigma)\right]} d \sigma .
$$

(Note that this assumes $\operatorname{det}\left[\begin{array}{lll}\mathbf{e}_{1}(t) & \mathbf{e}_{2}(t) & \mathbf{e}_{3}(t)\end{array}\right]>0$; if $\operatorname{det}\left[\begin{array}{lll}\mathbf{e}_{1}(t) & \mathbf{e}_{2}(t) & \mathbf{e}_{3}(t)\end{array}\right]$ $<0$, then $\alpha(t)$ can be reparametrized as $\alpha(-t)$ to reverse the sign. In particular, note that this assumption yields a preferred orientation for $\alpha$.)

If $\alpha$ is reparametrized by the inverse function $t\left(s_{\alpha}\right)$ as

$$
\alpha\left(s_{\alpha}\right)=\alpha\left(t\left(s_{\alpha}\right)\right)
$$

then the vectors

$$
\mathbf{e}_{1}\left(s_{\alpha}\right)=\alpha^{\prime}\left(s_{\alpha}\right), \quad \mathbf{e}_{2}\left(s_{\alpha}\right)=\alpha^{\prime \prime}\left(s_{\alpha}\right), \quad \mathbf{e}_{3}\left(s_{\alpha}\right)=\alpha^{\prime \prime \prime}\left(s_{\alpha}\right)
$$


form the affine Frenet frame of $\alpha$. The affine Frenet frame has the property that the matrix $\left[\begin{array}{lll}\mathbf{e}_{1}\left(s_{\alpha}\right) & \mathbf{e}_{2}\left(s_{\alpha}\right) & \mathbf{e}_{3}\left(s_{\alpha}\right)\end{array}\right]$ is an element of $S L(3)$, and the affine analog of the Frenet equations is

$$
\left[\begin{array}{lll}
\mathbf{e}_{1}^{\prime}\left(s_{\alpha}\right) & \mathbf{e}_{2}^{\prime}\left(s_{\alpha}\right) & \mathbf{e}_{3}^{\prime}\left(s_{\alpha}\right)
\end{array}\right]=\left[\begin{array}{lll}
\mathbf{e}_{1}\left(s_{\alpha}\right) & \mathbf{e}_{2}\left(s_{\alpha}\right) & \mathbf{e}_{3}\left(s_{\alpha}\right)
\end{array}\right]\left[\begin{array}{ccc}
0 & 0 & \kappa_{1}\left(s_{\alpha}\right) \\
1 & 0 & \kappa_{2}\left(s_{\alpha}\right) \\
0 & 1 & 0
\end{array}\right],
$$

where $\kappa_{1}\left(s_{\alpha}\right), \kappa_{2}\left(s_{\alpha}\right)$ are the affine curvature functions of $\alpha$. The affine arc length, affine Frenet frame, and affine curvatures are all invariant under the action of the equiaffine group.

Affine arc length is a very different notion from Euclidean arc length. Some of the differences include:

- Unlike Euclidean arc length, which depends only on the first derivative of $\alpha$, the affine arc length depends on the first three derivatives of $\alpha$. In general, this number is dependent on the dimension of the ambient affine space: the affine arc length of a curve $\alpha: I \rightarrow \mathbb{A}^{n}$ depends on the first $n$ derivatives of $\alpha$.

- The affine arc length is only nonzero for nondegenerate curves; so for instance, any curve contained in a plane in $\mathbb{A}^{3}$ has affine arc length zero according to this definition. It may, however, have nonzero affine arc length when regarded as a curve in $\mathbb{A}^{2}$.

It turns out that the affine arc length function of $\alpha$ can be expressed in terms of the Euclidean invariants of $\alpha$ :

Proposition 2.1. Let $\alpha: I \rightarrow \mathbb{R}^{3}$ be a nondegenerate curve. Let $\bar{s}(t), \kappa(t), \tau(t)$ be the Euclidean arc length, curvature, and torsion of $\alpha$, respectively, and suppose that $\tau(t)>0$. Then the affine arc length $s_{\alpha}(t)$ of $\alpha$ is given by

$$
s_{\alpha}(t)=\int_{0}^{t} \sqrt[6]{\kappa(\sigma)^{2} \tau(\sigma)} \bar{s}^{\prime}(\sigma) d \sigma .
$$

In particular, if $\alpha$ is parametrized by its Euclidean arc length $\bar{s}$, then

$$
s_{\alpha}(\bar{s})=\int_{0}^{\bar{s}} \sqrt[6]{\kappa(\sigma)^{2} \tau(\sigma)} d \sigma .
$$

Proof. Let $\left(\mathbf{e}_{1}(t), \mathbf{e}_{2}(t), \mathbf{e}_{3}(t)\right)$ denote the Euclidean Frenet frame of $\alpha$, and for convenience, let $v(t)$ denote $\bar{s}^{\prime}(t)$. Then we have

$$
\begin{aligned}
\alpha^{\prime}(t) & =v(t) \mathbf{e}_{1}(t) \\
\alpha^{\prime \prime}(t) & =v^{\prime}(t) \mathbf{e}_{1}(t)+v(t)^{2} \kappa(t) \mathbf{e}_{2}(t) \\
\alpha^{\prime \prime \prime}(t) & =\left(v^{\prime \prime}(t)-v(t)^{3} \kappa(t)^{2}\right) \mathbf{e}_{1}(t)+\left(3 v(t) v^{\prime}(t) \kappa(t)+v(t)^{2} \kappa^{\prime}(t)\right) \mathbf{e}_{2}(t) \\
& \quad+v(t)^{3} \kappa(t) \tau(t) \mathbf{e}_{3}(t)
\end{aligned}
$$

Therefore,

$$
\begin{aligned}
\sqrt[6]{\operatorname{det}\left[\alpha^{\prime}(t) \alpha^{\prime \prime}(t) \quad \alpha^{\prime \prime \prime}(t)\right]} & =\sqrt[6]{v(t)^{6} \kappa(t)^{2} \tau(t) \operatorname{det}\left[\mathbf{e}_{1}(t) \mathbf{e}_{2}(t) \mathbf{e}_{3}(t)\right]} \\
& =v(t) \sqrt[6]{\kappa(t)^{2} \tau(t)} \\
& =\sqrt[6]{\kappa(t)^{2} \tau(t)} \bar{s}^{\prime}(t)
\end{aligned}
$$

The result follows. 
Although the Euclidean quantities $\kappa(t), \tau(t), \bar{s}(t)$ are not invariant under the action of the equiaffine group, this proposition yields the following corollary:

Corollary 2.2. The Euclidean 1 -form $d s_{\alpha}=\sqrt[6]{\kappa^{2} \tau} d \bar{s}$ associated to a nondegenerate curve $\alpha: I \rightarrow \mathbb{R}^{3}$ is invariant under the action of the equiaffine group.

Remark 2.3. Since the Euclidean curvature and torsion are defined in terms of derivatives of $\alpha$ with respect to $\bar{s}$ and the affine curvatures are defined in terms of derivatives of $\alpha$ with respect to $s_{\alpha}$, in principle one could use equation (2.2) to express the affine curvatures in terms of the Euclidean curvature and torsion and their derivatives via straightfoward chain rule computations. However, these computations are rather messy and ultimately not very enlightening. While the Euclidean curvature and torsion are second- and third-order invariants, respectively, of $\alpha$, the affine curvatures $\kappa_{1}, \kappa_{2}$ are sixth- and fifth-order invariants, respectively, of $\alpha$. For details, see [7].

2.3. Surfaces and the affine first fundamental form. Let $U \subset \mathbb{R}^{2}$, and let $X: U \rightarrow \mathbb{R}^{3}$ be a parametrization of a regular surface $\Sigma$ (considered as a surface in either $\mathbb{E}^{3}$ or $\mathbb{A}^{3}$ ). In Euclidean geometry, one associates to $X$ the first fundamental form

$$
\mathrm{I}_{\mathrm{Euc}}=E d u^{2}+2 F d u d v+G d v^{2}
$$

where

$$
E=\left\langle X_{u}, X_{u}\right\rangle, \quad F=\left\langle X_{u}, X_{v}\right\rangle, \quad G=\left\langle X_{v}, X_{v}\right\rangle .
$$

The first fundamental form expresses the restriction of the Euclidean metric to $\Sigma$ as follows: for any tangent vector $\mathbf{v}$ to $\Sigma$, if we express $\mathbf{v}$ as

$$
\mathbf{v}=a X_{u}+b X_{v},
$$

then

$$
\langle\mathbf{v}, \mathbf{v}\rangle=\mathrm{I}_{\text {Euc }}(\mathbf{v})=E a^{2}+2 F a b+G b^{2} .
$$

Next, one associates to $\Sigma$ the second fundamental form

$$
\mathrm{II}_{\mathrm{Euc}}=e d u^{2}+2 f d u d v+g d v^{2},
$$

where

$$
e=\left\langle X_{u u}, N\right\rangle, \quad f=\left\langle X_{u v}, N\right\rangle, \quad g=\left\langle X_{v v}, N\right\rangle,
$$

and $N$ is a unit normal vector field to $\Sigma$. The second fundamental form encapsulates the curvature properties of the surface; in particular, the Gauss curvature of the surface is

$$
K=\frac{\operatorname{det}\left(\mathrm{II}_{\mathrm{Euc}}\right)}{\operatorname{det}\left(\mathrm{I}_{\mathrm{Euc}}\right)}=\frac{e g-f^{2}}{E G-F^{2}} .
$$

Now suppose that we consider $\Sigma$ as a surface in $\mathbb{A}^{3}$. There is no obvious analog to the Euclidean first fundamental form which is invariant under the action of the equiaffine group. However, we can construct a quadratic form which closely approximates the Euclidean second fundamental form: if we set

$$
\ell=\operatorname{det}\left[\begin{array}{lll}
X_{u} & X_{v} & X_{u u}
\end{array}\right], \quad m=\operatorname{det}\left[\begin{array}{lll}
X_{u} & X_{v} & X_{u v}
\end{array}\right], \quad n=\operatorname{det}\left[\begin{array}{lll}
X_{u} & X_{v} & X_{v v}
\end{array}\right],
$$

then the quadratic form

$$
\ell d u^{2}+2 m d u d v+n d v^{2}
$$


on the parametrized surface $X$ is invariant under the action of the equiaffine group. However, it is not quite invariant under a change of parametrization for $\Sigma$ : if we set

then we have

$$
\bar{X}(\bar{u}, \bar{v})=X(u(\bar{u}, \bar{v}), v(\bar{u}, \bar{v}))
$$

$$
\bar{\ell} d \bar{u}^{2}+\bar{m} d \bar{u} d \bar{v}+\bar{n} d \bar{v}^{2}=\left(\ell d u^{2}+2 m d u d v+n d v^{2}\right) J,
$$

where $J$ is the determinant of the Jacobian matrix of the coordinate transformation

$$
(\bar{u}, \bar{v}) \rightarrow(u(\bar{u}, \bar{v}), v(\bar{u}, \bar{v})) .
$$

This indeterminacy can be remedied as follows: it is straightforward to compute that

$$
\bar{\ell} \bar{n}-\bar{m}^{2}=\left(\ell n-m^{2}\right) J^{4} .
$$

Therefore, if $\ell n-m^{2} \neq 0$, then the quadratic form

$$
\mathrm{I}_{\mathrm{aff}}=\left|\ell n-m^{2}\right|^{-1 / 4}\left(\ell d u^{2}+2 m d u d v+n d v^{2}\right)
$$

is a well-defined, invariant quadratic form on $\Sigma$. This quadratic form is called the affine first fundamental form of $\Sigma$, and it can be used to define a metric on the surface $\Sigma$. Unlike in the Euclidean case, this metric is not necessarily positive definite; it may be positive or negative definite, or indefinite.

We make the following definitions (see 7]):

- A surface $\Sigma$ with parametrization $X: U \rightarrow \mathbb{A}^{3}$ is called nondegenerate if the quadratic form $\ell d u^{2}+2 m d u d v+n d v^{2}$ is nondegenerate (i.e., if $\left.\ell n-m^{2} \neq 0\right)$.

- A nondegenerate parametrized surface is called elliptic if the quadratic form $\mathrm{I}_{\text {aff }}$ is definite and hyperbolic if $\mathrm{I}_{\text {aff }}$ is indefinite.

Note that if $I_{\text {aff }}$ is negative definite, it can be made positive definite by interchanging the roles of $u$ and $v$. Thus, we will assume without loss of generality that $\mathrm{I}_{\text {aff }}$ is positive definite in the elliptic case.

As in the case of affine arc length, the affine first fundamental form can be expressed in terms of the Euclidean invariants of $\Sigma$ :

Proposition 2.4. Let $X: U \rightarrow \mathbb{R}^{3}$ be a regular parametrization for a nondegenerate surface $\Sigma$. Let $\mathrm{II}_{\mathrm{Euc}}$ denote the Euclidean second fundamental form of $\Sigma$, and let $K$ denote the Euclidean Gauss curvature of $\Sigma$. Then

$$
\mathrm{I}_{\mathrm{aff}}=|K|^{-1 / 4} \mathrm{II}_{\mathrm{Euc}} \text {. }
$$

Proof. Let $N$ be the Euclidean normal vector field to $\Sigma$. Then equations (2.3), (2.4) and standard properties of determinants imply that

$$
\begin{aligned}
& \ell=\operatorname{det}\left[\begin{array}{lll}
X_{u} & X_{v} & X_{u u}
\end{array}\right]=\operatorname{det}\left[\begin{array}{lll}
X_{u} & X_{v} & e N
\end{array}\right]=e \sqrt{E G-F^{2}}, \\
& m=\operatorname{det}\left[\begin{array}{llll}
X_{u} & X_{v} & X_{u v}
\end{array}\right]=\operatorname{det}\left[\begin{array}{llll}
X_{u} & X_{v} & f N
\end{array}\right]=f \sqrt{E G-F^{2}}, \\
& n=\operatorname{det}\left[\begin{array}{llll}
X_{u} & X_{v} & X_{v v}
\end{array}\right]=\operatorname{det}\left[\begin{array}{lll}
X_{u} & X_{v} & g N
\end{array}\right]=g \sqrt{E G-F^{2}} .
\end{aligned}
$$

Therefore, by equation (2.5),

$$
\begin{aligned}
\mathrm{I}_{\mathrm{aff}} & =\left|\ell n-m^{2}\right|^{-1 / 4}\left(\ell d u^{2}+2 m d u d v+n d v^{2}\right) \\
& =\left|e g-f^{2}\right|^{-1 / 4}\left|E G-F^{2}\right|^{1 / 4}\left(e d u^{2}+2 f d u d v+g d v^{2}\right) \\
& =|K|^{-1 / 4} \mathrm{II}_{\mathrm{Euc}} .
\end{aligned}
$$


Corollary 2.5. The Euclidean quadratic form $\mathrm{I}_{\mathrm{aff}}=|K|^{-1 / 4} \mathrm{II}_{\mathrm{Euc}}$ associated to a nondegenerate surface $\Sigma \subset \mathbb{R}^{3}$ is invariant under the action of the equiaffine group.

Remark 2.6. In addition to the affine first fundamental form $\mathrm{I}_{\text {aff }}$, one can associate to a nondegenerate surface $\Sigma \subset \mathbb{A}^{3}$ additional invariant structures, including an affine normal vector, a quadratic form $\mathrm{II}_{\mathrm{aff}}$ called the affine second fundamental form, and a cubic form $P$ called the Fubini-Pick form. Together, these form a complete set of invariants for nondegenerate surfaces in $\mathbb{A}^{3}$, in the sense that any two surfaces with the same first and second fundamental forms and Fubini-Pick forms must differ by an equiaffine transformation. See [7] for details.

\section{TwO ARC LENGTH FUNCTIONS FOR CURVES IN SURFACES}

Now suppose that $\alpha: I \rightarrow \mathbb{A}^{3}$ is a curve whose image is contained in a nondegenerate surface $\Sigma=X(U) \subset \mathbb{A}^{3}$. The restriction of $\mathrm{I}_{\text {aff }}$ to $\alpha$ defines an arc length function $s_{\Sigma}$ along $\alpha$ as follows:

$$
s_{\Sigma}(t)=\int_{0}^{t} \sqrt{\mathrm{I}_{\mathrm{aff}}\left(\alpha^{\prime}(\sigma)\right)} d \sigma
$$

We will refer to the function $s_{\Sigma}$ on $\alpha$ as the induced arc length function from $\Sigma$. Although the affine arc length $s_{\alpha}$ and the induced arc length $s_{\Sigma}$ are both "metric functions" along $\alpha$, in the sense that they allow us to measure the length of a curve embedded in an affine surface, they may or may not agree, even for fairly trivial examples.

Example 3.1. Let $\Sigma \subset \mathbb{R}^{3}$ be the unit sphere $S^{2}$, with the parametrization

$$
X(u, v)=[\cos (u) \cos (v), \sin (u) \cos (v), \sin (v)] .
$$

Regarded as a surface in $\mathbb{E}^{3}, \Sigma$ has uniform Gauss curvature $K=1$ and second fundamental form

$$
\mathrm{II}_{\mathrm{Euc}}=\cos ^{2}(v) d u^{2}+d v^{2}=\mathrm{I}_{\mathrm{Euc}} .
$$

(In fact, $\Sigma$ is the unique surface in $\mathbb{E}^{3}$ with the property that $\mathrm{II}_{\mathrm{Euc}}=\mathrm{I}_{\mathrm{Euc}}$.) Therefore, the affine first fundamental form of $\Sigma$ is

$$
\begin{aligned}
\mathrm{I}_{\mathrm{aff}} & =\left|K^{-1 / 4}\right| \mathrm{II}_{\mathrm{Euc}} \\
& =\cos ^{2}(v) d u^{2}+d v^{2} \\
& =\mathrm{I}_{\mathrm{Euc}} .
\end{aligned}
$$

Let $\alpha$ be a great circle on $\Sigma$, parametrized as

$$
\alpha(t)=X(t, 0)=[\cos (t), \sin (t), 0] .
$$

Because $\Sigma$ has the property that $\mathrm{II}_{\mathrm{Euc}}=\mathrm{I}_{\mathrm{Euc}}$, the induced arc length function $s_{\Sigma}(t)$ agrees with the Euclidean arc length function $\bar{s}(t)$; therefore,

$$
s_{\Sigma}(t)=t .
$$

But because $\alpha$ is contained in a plane, it is considered a degenerate curve in $\mathbb{A}^{3}$ and its affine arc length function $s_{\alpha}(t)$ is identically equal to zero.

For a less trivial example where the two arc lengths do not agree, consider the "spherical helix" curve

$$
\alpha(t)=X(8 t, t)=[\cos (8 t) \cos (t), \sin (8 t) \cos (t), \sin (t)]
$$


in $\Sigma$. Again, the induced arc length function $s_{\Sigma}(t)$ is equal to the Euclidean arc length function

$$
s_{\Sigma}(t)=\int_{0}^{t} \sqrt{1+64 \cos ^{2}(\sigma)} d \sigma
$$

while the affine arc length function $s_{\alpha}(t)$ is

$$
s_{\alpha}(t)=\int_{0}^{t} \sqrt[6]{48 \cos (\sigma)\left(43+672 \cos ^{2}(\sigma)\right)} d \sigma .
$$

The curve $\alpha$ along with graphs of the two arc length functions are shown in Figure1.
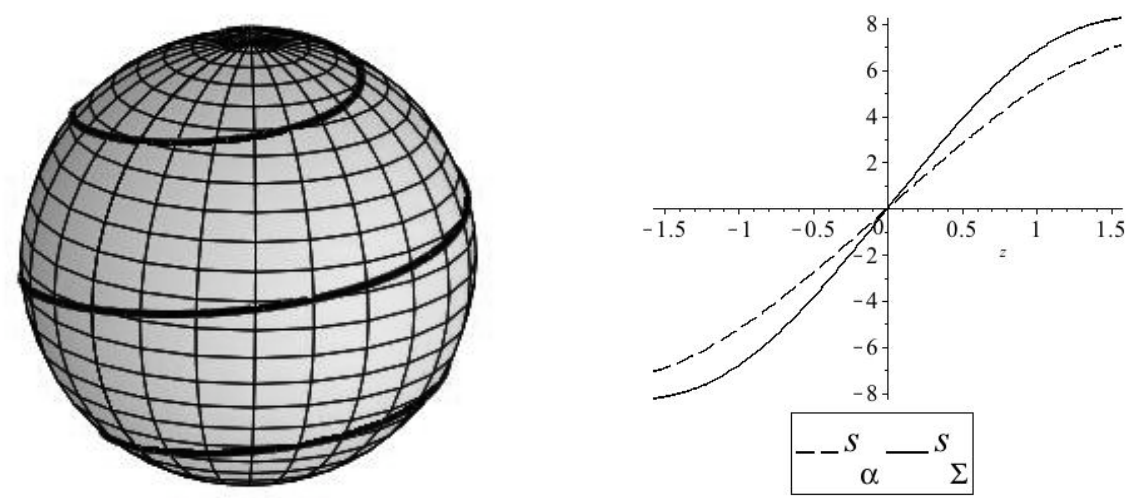

Figure 1. The spherical helix of Example 3.1

Example 3.2. Let $\Sigma \subset \mathbb{R}^{3}$ be the helicoid $H$, with the parametrization

$$
X(u, v)=[u \cos (v), u \sin (v), v] .
$$

Regarded as a surface in $\mathbb{E}^{3}, \Sigma$ has Gauss curvature $K=-\frac{1}{\left(u^{2}+1\right)^{2}}$ and second fundamental form

$$
\mathrm{II}_{\mathrm{Euc}}=-\frac{2}{\sqrt{u^{2}+1}} d u d v .
$$

Therefore, the affine first fundamental form of $\Sigma$ is

$$
\mathrm{I}_{\mathrm{aff}}=\left|K^{-1 / 4}\right| \mathrm{II}_{\mathrm{Euc}}=-2 d u d v .
$$

Let $\alpha$ be one of the rulings on $\Sigma$, parametrized as

$$
\alpha(t)=X\left(t, v_{0}\right)=\left[t \cos \left(v_{0}\right), t \sin \left(v_{0}\right), v_{0}\right],
$$

where $v_{0}$ is a constant. $\alpha(t)$ is a straight line; thus the vectors $\alpha^{\prime}, \alpha^{\prime \prime}, \alpha^{\prime \prime \prime}$ cannot be linearly independent, and the affine arc length $s_{\alpha}(t)$ is zero. Moreover, because $\alpha$ is an asymptotic curve in $\Sigma$, the restriction of the affine first fundamental form $\mathrm{I}_{\text {aff }}$ to $\alpha^{\prime}(t)$ is zero. Thus,

$$
s_{\alpha}(t)=s_{\Sigma}(t)=0,
$$

and the two (degenerate) arc length functions on $\alpha$ coincide.

For a nondegenerate example where the two arc lengths do not agree, consider the "helical spiral" curve

$$
\alpha(t)=X(t, \pi t)=[t \cos (\pi t), t \sin (\pi t), \pi t]
$$


in $H$. The induced arc length function is

$$
s_{\Sigma}(t)=\int_{0}^{t} \sqrt{2 \pi} d \sigma=\sqrt{2 \pi} t,
$$

while the affine arc length function is

$$
s_{\alpha}(t)=\int_{0}^{t}\left(6 \pi^{4}+\sigma^{2} \pi^{6}\right)^{1 / 6} d \sigma .
$$

These functions are qualitatively quite different: $s_{\Sigma}$ is clearly linear in $t$, while $s_{\alpha} \sim \pi t^{4 / 3}$. The curve $\alpha$ along with graphs of the two arc length functions are shown in Figure 2,
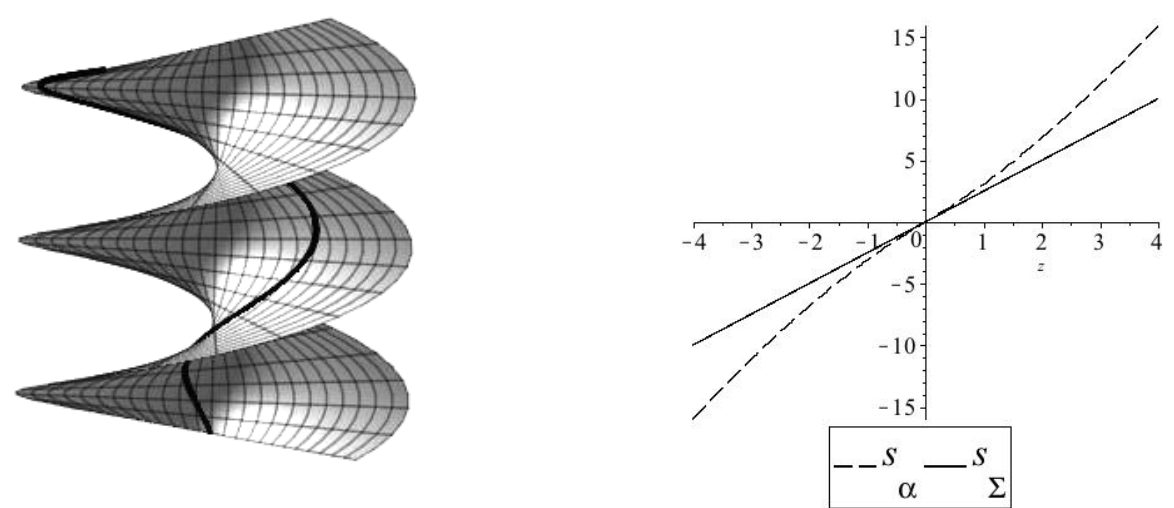

Figure 2. The helical spiral of Example 3.2

These examples raise the question: given a surface $\Sigma \subset \mathbb{A}^{3}$, are there nondegenerate curves in $\Sigma$ for which the two arc length functions $s_{\alpha}(t), s_{\Sigma}(t)$ coincide? We will consider this question in the following section.

\section{MAIN THEOREM}

In this section, we explore the question of when the affine arc length function $s_{\alpha}$ and the induced arc length function $s_{\Sigma}$ for a curve $\alpha \subset \Sigma \subset \mathbb{A}^{3}$ are equal. This question motivates the following definition.

Definition 4.1. A nondegenerate curve $\alpha$ contained in a regular, nondegenerate surface $\Sigma \subset \mathbb{A}^{3}$ will be called commensurate if the affine arc length function $s_{\alpha}$ and the induced arc length function $s_{\Sigma}$ associated to $\alpha$ are equal.

We have seen in $\$ 3$ that there exist examples of both commensurate and noncommensurate curves.

Theorem 4.2. Let $X: U \rightarrow \mathbb{R}^{3}$ be a regular parametrization for a nondegenerate surface $\Sigma$, and let $\alpha: I \rightarrow \mathbb{R}^{3}$ be a regular, nondegenerate curve contained in $\Sigma$. Then $\alpha$ is a commensurate curve if and only if, for all $t \in I$,

$$
\operatorname{det}\left[\begin{array}{lll}
\alpha^{\prime}(t) & \alpha^{\prime \prime}(t) & \alpha^{\prime \prime \prime}(t)
\end{array}\right]=\left[\mathrm{I}_{\mathrm{aff}}\left(\alpha^{\prime}(t)\right)\right]^{3} .
$$


Proof. From equations (3.1) and (2.6), we know that, along $\alpha$,

$$
s_{\Sigma}(t)=\int_{0}^{t} \sqrt{\mathrm{I}_{\mathrm{aff}}\left(\alpha^{\prime}(\sigma)\right)} d \sigma .
$$

Similarly, from equation (2.1), we know that

$$
s_{\alpha}(t)=\int_{0}^{t} \sqrt[6]{\operatorname{det}\left[\alpha^{\prime}(\sigma) \alpha^{\prime \prime}(\sigma) \alpha^{\prime \prime \prime}(\sigma)\right]} d \sigma .
$$

The curve $\alpha$ is commensurate if and only if, for all $t \in I, s_{\alpha}(t)=s_{\Sigma}(t)$, i.e., if and only if

$$
\int_{0}^{t} \sqrt[6]{\operatorname{det}\left[\alpha^{\prime}(\sigma) \alpha^{\prime \prime}(\sigma) \alpha^{\prime \prime \prime}(\sigma)\right]} d \sigma=\int_{0}^{t} \sqrt{\mathrm{I}_{\mathrm{aff}}\left(\alpha^{\prime}(\sigma)\right)} d \sigma .
$$

This equation holds for all $t$ if and only if the integrands are equal, i.e., if

$$
\begin{aligned}
& \sqrt[6]{\operatorname{det}\left[\alpha^{\prime}(t) \alpha^{\prime \prime}(t) \alpha^{\prime \prime \prime}(t)\right]}=\sqrt{\mathrm{I}_{\mathrm{aff}}\left(\alpha^{\prime}(t)\right)} \\
& \Leftrightarrow \quad \operatorname{det}\left[\begin{array}{lll}
\alpha^{\prime}(t) & \alpha^{\prime \prime}(t) & \alpha^{\prime \prime \prime}(t)
\end{array}\right]=\left[\mathrm{I}_{\mathrm{aff}}\left(\alpha^{\prime}(t)\right)\right]^{3} .
\end{aligned}
$$

Thus, $\alpha$ is commensurate if and only if

$$
\operatorname{det}\left[\begin{array}{lll}
\alpha^{\prime}(t) & \alpha^{\prime \prime}(t) & \alpha^{\prime \prime \prime}(t)
\end{array}\right]=\left[\mathrm{I}_{\mathrm{aff}}\left(\alpha^{\prime}(t)\right)\right]^{3} .
$$

Remark 4.3. Expressing the condition of the theorem by equation (4.1) allows us to relax the assumption that both sides must be positive. This is useful when the quadratic form $\mathrm{I}_{\text {aff }}$ is indefinite and the right-hand side may take on negative values.

We can express the condition (4.1) in terms of the Euclidean invariants of $\alpha$ and $\Sigma:$

Corollary 4.4. Let $X: U \rightarrow \mathbb{R}^{3}$ be a regular parametrization for a nondegenerate surface $\Sigma$, and let $\alpha: I \rightarrow \mathbb{R}^{3}$ be a regular, nondegenerate curve contained in $\Sigma$. Then $\alpha$ is a commensurate curve if and only if, for all $t \in I$,

$$
\kappa(t)^{2} \tau(t)=\left(|K(t)|^{-1 / 4} k_{n}(t)\right)^{3},
$$

where $\kappa(t), \tau(t)$ are the Euclidean curvature and torsion functions of $\alpha, K(t)$ is the Gauss curvature of $\Sigma$ at the point $\alpha(t)$, and $k_{n}(t)$ is the normal curvature of $\Sigma$ at the point $\alpha(t)$ in the direction of $\alpha^{\prime}(t)$.

Proof. By Proposition 2.1, the left-hand side of (4.1) is equal to

$$
\kappa(t)^{2} \tau(t)\left\|\alpha^{\prime}(t)\right\|^{6} \text {. }
$$

By Proposition 2.4 and the fact that normal curvature is defined by

$$
k_{n}(t)=\frac{1}{\left\|\alpha^{\prime}(t)\right\|^{2}} \mathrm{I}_{\mathrm{Euc}}\left(\alpha^{\prime}(t)\right),
$$

the right-hand side of (4.1) is equal to

$$
\begin{aligned}
\left(|K(t)|^{-1 / 4} \operatorname{II}_{\mathrm{Euc}}\left(\alpha^{\prime}(t)\right)\right)^{3} & =\left(|K(t)|^{-1 / 4} k_{n}(t)\left\|\alpha^{\prime}(t)\right\|^{2}\right)^{3} \\
& =\left(|K(t)|^{-1 / 4} k_{n}(t)\right)^{3}\left\|\alpha^{\prime}(t)\right\|^{6} .
\end{aligned}
$$


Since $\alpha$ is assumed to be regular, and hence $\left\|\alpha^{\prime}(t)\right\| \neq 0$, it follows that

$$
\kappa(t)^{2} \tau(t)=\left(|K(t)|^{-1 / 4} k_{n}(t)\right)^{3} .
$$

Theorem 4.2 not only gives us a condition on when a curve is commensurate, it also guarantees the existence of commensurate curves on any nondegenerate surface $\Sigma$.

Corollary 4.5. Let $X: U \rightarrow \mathbb{R}^{3}$ be a regular parametrization for a nondegenerate surface $\Sigma$. Given any point $\mathbf{x} \in \Sigma$ and any tangent vector $\mathbf{v} \in T_{\mathbf{x}} \Sigma$ for which $\mathrm{I}_{\mathrm{aff}}(\mathbf{v}) \neq 0$, there exists a 1-parameter family of commensurate curves $\alpha$ in $\Sigma$ such that $\alpha(0)=\mathbf{x}$ and $\alpha^{\prime}(0)=\mathbf{v}$.

Remark 4.6. The hypothesis $\mathrm{I}_{\mathrm{aff}}(\mathbf{v}) \neq 0$ is not strictly necessary if we extend our definitions to degenerate curves in the obvious way.

Proof. Let $\mathbf{x}=X\left(u_{0}, v_{0}\right)$ and $\mathbf{v}=a X_{u}+b X_{v}$. We can write

$$
\alpha(t)=X(u(t), v(t))
$$

for some smooth functions $u(t), v(t)$. The condition (4.1) is invariant under reparametrizations of $\alpha$, so without loss of generality we may assume (locally) that $u(t)=$ $u_{0}+a t$, and therefore

$$
\alpha(t)=X\left(u_{0}+a t, v(t)\right) .
$$

Equation (4.1) then becomes a 3rd-order nonlinear ODE for the function $v(t)$. The conditions $\alpha(0)=\mathbf{x}, \alpha^{\prime}(0)=\mathbf{v}$ are equivalent to the initial conditions

$$
v(0)=v_{0}, \quad v^{\prime}(0)=b
$$

for the function $v(t)$. The local existence/uniqueness theorem for ODEs guarantees that for any real number $c$, there exists a unique local solution to (4.1) with

$$
v(0)=v_{0}, \quad v^{\prime}(0)=b, \quad v^{\prime \prime}(0)=c .
$$

For example, if we set $u_{0}=0, a=1$, so that

$$
\alpha(t)=X(t, v(t))
$$

then equation (4.1) becomes

$$
\operatorname{det}\left[\frac{d}{d t} X(t, v(t)) \frac{d^{2}}{d t^{2}} X(t, v(t)) \frac{d^{3}}{d t^{3}} X(t, v(t))\right]=\left[\mathrm{I}_{\mathrm{aff}}\left(\frac{d}{d t} X(t, v(t))\right)\right]^{3} .
$$

For most surfaces $X$, equation (4.3) is highly nonlinear and cannot be solved explicitly for $v(t)$. However, we can often numerically solve for commensurate curves and examine them qualitatively. In \$5 we will use this process to compute examples of commensurate curves on various surfaces. 


\section{EXAMPLES}

Example 5.1. Let $\Sigma \subset \mathbb{R}^{3}$ be the unit sphere, and let $\alpha$ be a commensurate curve on $\Sigma$. For simplicity, assume that $\alpha$ is parametrized by its Euclidean arc length $\bar{s}$. Since all normal curvatures on $\Sigma$ are equal to 1, Corollary 4.4 implies that the curve $\alpha(\bar{s})$ on $\Sigma$ is commensurate if and only if its curvature and torsion satisfy

$$
\kappa(\bar{s})^{2} \tau(\bar{s}) \equiv 1 .
$$

Moreover, the fact that $\alpha$ lies on the unit sphere implies that

$$
\left(\frac{1}{\kappa(\bar{s})}\right)^{2}+\left(\frac{1}{\tau(\bar{s})} \frac{d}{d s}\left(\frac{1}{\kappa(\bar{s})}\right)\right)^{2}=1 .
$$

(See Exercise 1.3.24 of [6].) Together, these two equations imply that $\kappa(\bar{s})$ satisfies the ODE

$$
\left(\kappa^{\prime}(\bar{s})\right)^{2}=\frac{\kappa(\bar{s})^{2}-1}{\kappa(\bar{s})^{2}} .
$$

The general solution of this equation is

$$
\kappa(\bar{s})= \pm \sqrt{(\bar{s}+c)^{2}+1}
$$

where $c \in \mathbb{R}$. Since $\kappa(\bar{s})$ is assumed to be positive and $\bar{s}$ is only well-defined up to an additive constant, we may assume without loss of generality that

$$
\kappa(\bar{s})=\sqrt{\bar{s}^{2}+1},
$$

and then equation (5.1) implies that

$$
\tau(\bar{s})=\frac{1}{\bar{s}^{2}+1} .
$$

Unfortunately, the corresponding Frenet equations cannot be integrated analytically, but we can integrate them numerically to obtain the curve shown in Figure 3 . Every other commensurate curve on the sphere can be obtained by translating and rotating this one.
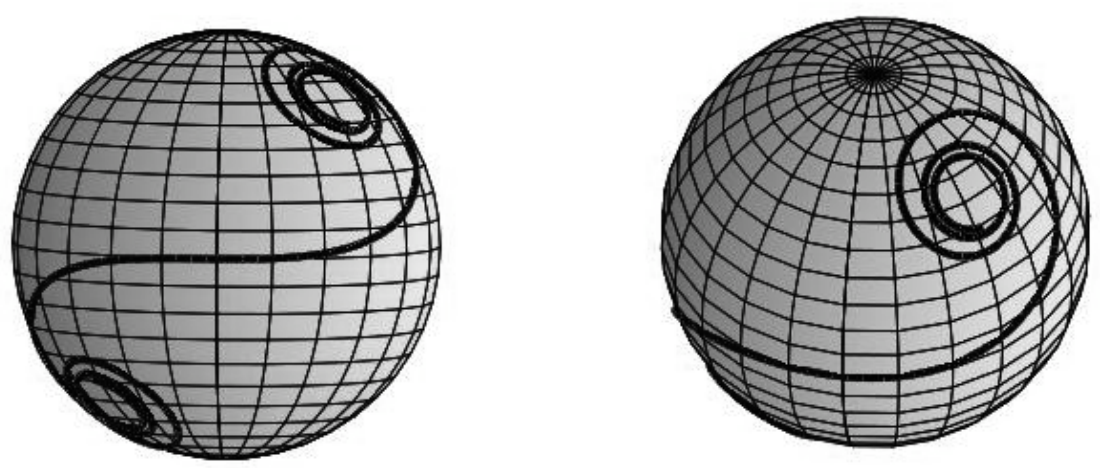

Figure 3. Two views of a commensurate curve on the sphere 
Remark 5.2. Because all normal curvatures on the unit sphere are equal to 1 and the commensurate curves have curvature function $\kappa(\bar{s})=\sqrt{\bar{s}^{2}+1}$, they have geodesic curvature function $\kappa_{g}(\bar{s})=\bar{s}$. Thus the commensurate curves are the spherical analogs of plane curves with curvature $\kappa(\bar{s})=\bar{s}$. These plane curves are called Euler spirals or clothoid curves (see, e.g., [1]), and they have a long and interesting history. They first appeared as the solution to an elasticity problem posed in 1694 by James Bernoulli [2, then in work of Augustin Fresnel in 1816 regarding the problem of light diffracting through a slit 4, and again in work of Arthur Talbot in 1901 related to designing railroad tracks so as to provide as smooth a riding experience as possible [8]. A nice account of the history of Euler spirals is given in [5].

For the remaining examples in this section, we computed commensurate curves as follows. Example 5.1 illustrates how the local parametrization (4.2) may be too limiting, as it assumes that the curve is never tangent to the $v$-parameter curves of $\Sigma$ and so may only be accurate for computing small segments of the curve. In order to remedy this weakness, for the remaining examples we assume that the curve is parametrized as

$$
\alpha(t)=X(u(t), v(t))
$$

where

$$
u^{\prime}(t)=\cos (\theta(t)), \quad v^{\prime}(t)=\sin (\theta(t))
$$

for some unknown function $\theta(t)$. Then, for a given parametrization $X(u, v)$ of $\Sigma$, equation (4.1) becomes a second-order ODE for the function $\theta(t)$, with coefficients depending on the functions $u(t), v(t)$. We used the Rosenbrock stiff algorithm in MAPLE 15 to numerically solve the system consisting of this ODE together with equations (5.2) for various choices of initial conditions in order to generate the curves in the following examples.

Example 5.3. Let $\Sigma \subset \mathbb{R}^{3}$ be a paraboloid, parametrized via a Monge patch in polar coordinates as

$$
X(u, v)=\left[v \cos (u), v \sin (u), v^{2}\right] .
$$

Some commensurate curves on $\Sigma$ are shown in Figure 4, As one might expect since

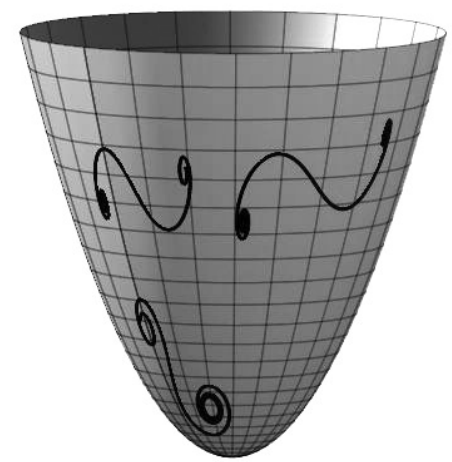

Figure 4. Commensurate curves on the paraboloid 
the paraboloid is an elliptic surface, these curves appear qualitatively similar to the commensurate curves on the sphere.

In our next three examples, we compute some examples of nondegenerate commensurate curves on hyperbolic surfaces. Such curves can never be tangent to an asymptotic direction, since these are precisely the null directions for $\mathrm{I}_{\text {aff }}$. (If an asymptotic curve happens to be contained in a plane, as is the case for any straight line contained in a surface, then the curve is technically commensurate, but then it is also degenerate.) Experimentally, we observe in all three cases that commensurate curves tend to approach asymptotic tangent directions fairly quickly, and that the numerical integration algorithm breaks down when the tangent vector to the curve gets too close to an asymptotic direction.

Example 5.4. Let $\Sigma \subset \mathbb{R}^{3}$ be a hyperbolic paraboloid, parametrized as

$$
X(u, v)=[u, v, u v],
$$

so that the coordinate curves are precisely the two families of straight lines in $\Sigma$. Some commensurate curves on $\Sigma$ are shown in Figure 5 .

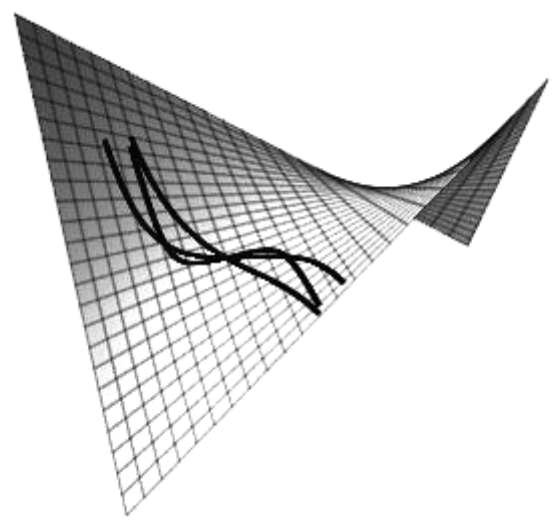

Figure 5. Commensurate curves on the hyperbolic paraboloid

Example 5.5. Let $\Sigma \subset \mathbb{R}^{3}$ be a hyperboloid, parametrized as

$$
X(u, v)=[\cos (u)-v \sin (u), \sin (u)+v \cos (u), v],
$$

so that the $v$ coordinate curves are one of the two families of straight lines in $\Sigma$. Some commensurate curves on $\Sigma$ are shown in Figure 6 .

Example 5.6. Let $\Sigma \subset \mathbb{R}^{3}$ be a helicoid, parametrized as

$$
X(u, v)=[u \cos (v), u \sin (v), v] .
$$

Some commensurate curves on $\Sigma$ are shown in Figure 7. 


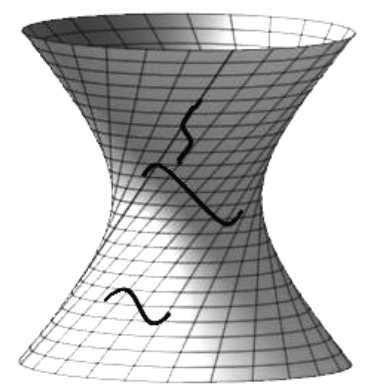

Figure 6. Commensurate curves on the hyperboloid
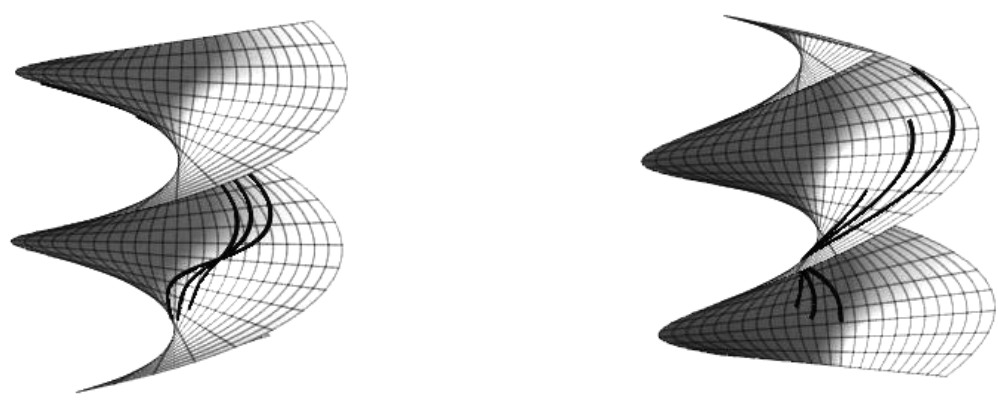

FiguRE 7. Commensurate curves on the helicoid

\section{Conclusion}

While the affine arc length of a curve $\alpha$ in $\mathbb{A}^{3}$ and the affine first fundamental form of a surface $\Sigma \subset \mathbb{A}^{3}$ are both regarded as "metrics" on these objects in a certain sense, they are clearly very different notions from their Euclidean analogs. In particular, the fact that they generally give rise to different length functions on a curve contained in a surface is a radical departure from Euclidean geometry. Nevertheless, Corollary 4.5 guarantees the existence of a large family of commensurate curves on any nondegenerate surface, and it seems natural to investigate these curves to see whether they have any other properties of interest. While we are not aware of any immediate practical applications for such curves in general, the connection with Euler spirals in the case where $\Sigma$ is a sphere (as described in Remark 5.2) is tantalizing and suggests that these curves might be of more general interest. Unfortunately the differential equation (4.1) describing these curves is highly nonlinear, and therefore any rigorous analysis of its solutions presents a formidable challenge. 


\section{REFERENCES}

[1] Milton Abramowitz and Irene A. Stegun, Handbook of mathematical functions with formulas, graphs, and mathematical tables, National Bureau of Standards Applied Mathematics Series, vol. 55, For sale by the Superintendent of Documents, U.S. Government Printing Office, Washington, D.C., 1964. MR.0167642 (29 \#4914)

[2] James Bernoulli, Curvatura laminae elasticae, Acta eruditorum Lipsiae (1694), 262-276.

[3] Manfredo P. do Carmo, Differential geometry of curves and surfaces, Prentice-Hall Inc., Englewood Cliffs, N.J., 1976. Translated from the Portuguese. MR0394451 (52 \#15253)

[4] Augustin Fresnel, Mémoire sur la diffraction de la lumière, où l'on examine particulièrement le phénomène des franges coloréesque présententles ombres des corps éclairés par un point lumineux, Ann. Phys. Chimie 1 (1816), 239-281.

[5] Raph Levien, The elastica: a mathematical history, http://www.levien.com/phd/ elastica_hist.pdf.

[6] John Oprea, Differential geometry and its applications, 2nd ed., Classroom Resource Materials Series, Mathematical Association of America, Washington, DC, 2007. MR2327126 (2008k:53002)

[7] Bu Chin Su, Affine differential geometry, Science Press, Beijing, 1983. MR724783 (85g:53010)

[8] Arthur Talbott, The railway transition spiral, McGraw-Hill, New York, 1901.

Department of Mathematics, 395 UCB, University of Colorado, Boulder, Colorado 80309-0395

E-mail address: Jeanne.Clelland@colorado.edu

Department of Physics, 390 UCB, University of Colorado, Boulder, Colorado 803090390

E-mail address: Edward.Estrada@colorado.edu

Department of Physics, 390 UCB, University of Colorado, Boulder, Colorado 803090390

E-mail address: Molly.May@colorado.edu

Department of Physics, 390 UCB, University of Colorado, Boulder, Colorado 803090390

Current address: Department of Physics, University of Guelph, Guelph, ON N1G 2W1, Canada

E-mail address: jmille16@uoguelph.ca

Department of Physics, 390 UCB, University of Colorado, Boulder, Colorado 803090390

Current address: USN, 404 Brookfield Lane, Goose Creek, South Carolina 29445

E-mail address: peneyra.s@gmail.com 0390

Department of Physics, 390 UCB, University of Colorado, Boulder, Colorado 80309-

Current address: The Fulton School, 123 Schoolhouse Road, St. Albans, Missouri 63073

E-mail address: schmidmt@gmail.com 